\title{
PROCESSO DE VERTICALIZAÇÃO NA CENTRAL DE DISTRIBUIÇÃO: UM ESTUDO DE CASO NUMA EMPRESA DE FABRICAÇÃO E ARMAZENAMENTO DE BALDES PLÁSTICOS
}

Adriano Pereira da Silva ${ }^{(1)}$ (pereiras.adriano@gmail.com), Francisco Gaudêncio Mendonça Freires ${ }^{(2)}$ (gaudenciof@yahoo.com)

\footnotetext{
(1) Universidade Federal da Bahia (UFBA); Programa de Engenharia Industrial

(2) Universidade Federal da Bahia (UFBA); Departamento de Engenharia Mecânica
}

RESUMO: Este trabalho apresenta um projeto de verticalização do estoque de um centro de distribuição, sendo caracterizada pelo estudo numa empresa de embalagens plásticas, na qual impacta positivamente no processo como um todo, em uma melhor relação com os clientes, demostrando uma melhor eficiência para o comprimento dos prazos e acima de tudo o compromisso de buscar soluções inovadoras e assim, na melhoria continua dos processos operacionais, controle de estoque face às demandas dos clientes e principalmente eliminação dos desperdícios de armazenamento. Para tanto, observa-se como objetivo geral a implantação e consolidação da verticalização na central de distribuição logística. Em paralelo, os objetivos específicos se caracterizam em: identificar meio de manter o inventário atualizado; promover a qualificação dos profissionais; e promover aumento da produtividade, assegurando o atendimento dos prazos. Diante disso, apresenta-se as etapas de execução do plano de trabalho para chegada do propósito de otimização do espaço físico e potencialização do nível de operação.

PALAVRAS-CHAVE: VERTICALIZAÇÃO, CENTRO DE DISTRIBUIÇÃO, ARMAZENAMENTO.

\section{VERTICALIZATION PROCESS IN THE DISTRIBUTION CENTER: A CASE IN A PLASTIC BUCKET MANUFACTURING AND STORAGE COMPANY}

ABSTRACT: This paper presents a project of verticalization of the stock of a distribution center, characterized by the study in a plastic packaging company, which positively impacts the process as a whole, in a better relationship with customers, demonstrating a better efficiency for the length of deadlines and above all the commitment to seek innovative solutions and thus, the continuous improvement of operational processes, inventory control against customer demands and especially elimination of storage waste. To this end, the general objective is the implementation and consolidation of vertical integration in the logistics distribution center. In parallel, the specific objectives are: to identify means of keeping inventory updated; promote the qualification of professionals; and promote increased productivity, ensuring deadlines are met. Given this, we present the steps of execution of the work plan to arrive at the purpose of optimizing the physical space and enhancing the level of operation.

KEYWORDS: VERTICALIZATION, DISTRIBUTION CENTER, STORAGE. 


\section{INTRODUÇÃO}

O Centro de Distribuição é uma configuração de armazém na qual são recebidas cargas consolidadas de diversos fornecedores. Essas cargas são fracionadas a fim de agrupar os produtos em quantidade e sortimento corretos, assim, realizando o escoamento destes itens. A relação entre todas as funções de uma Central de Distribuição está relacionada com a mercadoria que chega do fornecedor e é recebida pelo CD, podendo ser armazenada para futura expedição ou pode ser diretamente encaminhada para expedição. Assim, o mínimo de tempo possível a fim de não manter estoque é quando destinada à armazenagem, a mercadoria é movimentada até o seu devido local no estoque, até que seja solicitada em um determinado pedido, então separada e encaminhada para expedição, onde será transportada até o destino adequado. (APTE; VISWANATHAN, 2000).

Tomando como base o gerenciamento da cadeia produtiva, foi desenvolvido um projeto de verticalização de estoque representa para a organização uma inovação em todo conceito de logística e na forma como o trabalho se adequará da melhor forma possível para satisfazer na mais alta qualidade os clientes, com agilidade de separação da carga, aumento da produtividade, maior competividade em relação aos concorrentes e redução do tempo de entrega ao cliente final. Por conseguinte, o problema proposto se refere a: Como aumentar o espaço de armazenamento (aproveitamento do espaço físico), otimizando a distribuição do produto acabado? Como hipótese, pode-se identificar a criação de mecanismos de coleta e análise de dados, instalação de sistema de informação e reconfiguração do layout.

Dentro dessa perspectiva, o objetivo geral do presente trabalho se refere à implantação e consolidação da verticalização na central de distribuição logística. Em paralelo, os objetivos específicos se caracterizam em: identificar meio de manter o inventário atualizado; Promover a qualificação dos profissionais; E promover aumento da produtividade, assegurando o atendimento dos prazos.

Nesse contexto, serão realizadas análises do processo de transferência de fábrica para o centro de distribuição, identificando ganhos, formas de agilizar o processo, além da eliminação dos desperdícios; redesenho dos processos de transferência externa, recebimento, conferencia, armazenagem, transferência interna e expedição; prevê o desenho do processo de paletização; definição dos indicadores operações aplicáveis ao processo; capacitação e treinamento de novos processos operacionais; simulação dos processos operacionais e stress test; E acompanhamento operacional através dos parâmetros definidos e verificados. 


\section{REFERENCIAL TEÓRICO}

A fundamentação teórica permite verificar o estado do problema a ser pesquisado, sob o aspecto teórico e de outros estudos e pesquisas já realizados. Assim, sustenta à coleta e análise dos dados e apresenta os conceitos, teorias e modelos que irão sustentar a argumentação que se segue.

\subsection{Central de distribuição}

As operações dentro da Central de Distribuição são caracterizadas por diversas etapas. Assim, inicia-se pelo recebimento, que se remete a primeira etapa da trajetória do produto no CD. Essa etapa é essencial para a realização das outras atividades, envolvendo o descarregamento das cargas e a conferência da quantidade e da qualidade dos produtos entregues pelos fornecedores. Após registrar os produtos, o sistema de gerenciamento do armazém indica o endereço na área de armazenagem ou em outras áreas organizacionais onde os produtos deverão ser alocados.

Por conseguinte, a movimentação interna dos produtos é o transporte de pequenas quantidades de produtos no armazém. Invariavelmente, a movimentação e o manuseio de materiais absorvem tempo, mão-de-obra e dinheiro. Assim, é preciso minimizar o manuseio dos materiais, a fim de não provocar movimentos desnecessários, além de aumentar o risco de dano ou perda do produto. A oportunidade de reduzir a intensidade da mão-de-obra e aumentar sua produtividade reside nas novas tecnologias de movimentação e manuseio de materiais que estão emergindo atualmente. Segundo Moura (2008), o tipo de equipamento utilizado na movimentação de materiais afeta a eficiência e o custo de operação do CD. A armazenagem é a guarda temporária de produtos para posterior distribuição. Os estoques são necessários para o equilíbrio entre a demanda e a oferta. No entanto, as empresas visam manter níveis de estoques baixos, pois estes geram custos elevados.

A área de armazenagem dos CDs é composta, segundo Calazans (2012), por estruturas como porta-paletes, drive-in, estantes e racks, que são separadas por corredores para ter acesso às mercadorias. Esses corredores são sinalizados para facilitar a operação do CD. Separação de pedidos A separação de pedidos (picking) é a "coleta do mix correto de produtos, em suas quantidades corretas da área de armazenagem para satisfazer as necessidades do consumidor" (LIMA, 2012). É uma etapa fundamental do ciclo do pedido, pois consome cerca de $60 \%$ dos custos operacionais de um CD (TOMPKINS, 1996).

A área de estocagem na maioria dos armazéns ocupa um grande espaço, devido ao 
acondicionamento dos estoques. Assim, a separação de pedidos, que é realizada nessa área, implica em grandes deslocamentos por parte dos operadores. No entanto, existem algumas alternativas intermediárias, segundo Lima (2012), para diminuir esse tempo gasto com o deslocamento, como: algoritmos para definição das rotas de coleta, lógicas de endereçamento e métodos alternativos de organização do trabalho.

Expedição é a última etapa a ser realizada no CD. Consiste basicamente na verificação e no carregamento dos produtos nos veículos, podendo envolver algumas atividades como: conferência do pedido, preparação dos documentos de expedição e pesagem da carga para determinação do custo de transporte.

A partir destes requisitos, a gestão dos estoques no curso da cadeia de suprimentos é essencial para a administração eficiente dos materiais nas organizações, sejam estas públicas ou privadas. A falta de materiais de alta rotatividade, ou mesmo de baixa, porém que sejam importantes para o funcionamento da organização, pode implicar significativos prejuízos para as organizações, uma vez que podem ter, devido a estas faltas, seu sistema produtivo afetado. A escolha de uma ferramenta eficiente para auxiliar os gestores de almoxarifados na administração de materiais pode ajudar a solucionar diferentes problemas que esse setor organizacional vem enfrentando (GIANESI; BIAZZI, 2011).

A implantação do projeto impacta positivamente no processo como um todo, na qual, através de KPI (Key Performance Indicator) revela o nível de evolução do projeto, possibilitando, assim, uma melhor relação com os clientes, demonstrando uma melhor eficiência para o atendimento ao consumidor final. Em nível comercial, representa maior velocidade, agilidade na entrega do produto, proporcionando um aumento na relação da confiança e segurança para o mercado e fortalecendo as parcerias assim com expandido o negócio.

\subsection{Sistema Kanban de abastecimento}

A partir da observação do sistema tradicional de abastecimento, percebe-se o estoque empurrando a produção, ou seja, dependendo da quantidade de peças ao lado do montador, a velocidade da produção aumenta ou diminui (MOURA, 1996). O controle de estoques se torna mais difícil, e para compensar este fato utilizam-se estoques de segurança maiores, que geralmente são recebidos em grandes lotes de uma só vez. Isto gera a necessidade de uma grande área física destinada ao armazenamento do material.

A velocidade do abastecimento de materiais não acontece de forma totalmente sincronizada com o consumo real das linhas de montagem, isto frequentemente provoca a sobra ou 
a falta de material. Assim, de acordo com Moura, Umeda (1996), O Kanban é uma técnica japonesa de gestão de materiais e de produção no momento exato (Just-in-Time), que é controlado através do movimento de cartão (Kanban). A inspiração inicial para o desenvolvimento do Kanban, foi a análise sobre o sistema de funcionamento dos supermercados americanos. LIMA (2012), destaca que do supermercado se pega à ideia de visualizar o processo inicial numa linha de produção como um tipo de loja. O processo final (cliente) vai até o processo inicial (supermercado) para adquirir as peças necessárias (gêneros) no momento e na quantidade que precisa. O processo inicial imediatamente produz a quantidade recém retirada (abastecimento das prateleiras).

Assim, o sistema Kanban é caracterizado ainda pelo uso de cartões que funcionam como sinalizadores dentro de um processo de produção. Funciona de modo que um cartão sinaliza para o processo anterior o seu status (situação) atual. A finalidade do cartão Kanban é puxar a produção, ou seja, a linha de montagem final sinaliza a sua necessidade e o processo anterior produz exatamente o que é necessário (TOMPKINS, 1996).

\subsection{Sistema de gerenciamento de armazém}

Nos dias de hoje, com o ambiente empresarial cada vez mais competitivo, a tecnologia de informação, quando bem utilizada, torna-se um forte diferencial entre as empresas pela busca na excelência do atendimento ao cliente. Dessa forma, cada vez mais as empresas buscam alternativas para facilitar o gerenciamento de suas atividades, visando aumentar o controle e obter informações precisas que possam de fato agilizar a tomada de decisões e, consequentemente, melhorar o nível de serviço prestado (TOMPKINS, 1996).

De acordo com Lima (2012), um WMS (Warehouse Management System) é um sistema de gestão por software que melhora as operações do armazém, através do eficiente gerenciamento de informações e conclusão das tarefas, com um alto nível de controle e acuracidade do inventário. Segundo o referido autor, as informações gerenciadas são originadas de transportadoras, fabricantes, sistemas de informações de negócios, clientes e fornecedores. O WMS utiliza estas informações para receber, inspecionar, estocar, separar, embalar e expedir mercadorias da forma mais eficiente. A eficiência é obtida através do planejamento, roteirização e tarefas múltiplas dos diversos processos do armazém. Dessa forma, os sistemas de informação funcionam como elos que ligam as atividades logísticas em um processo integrado e têm-se mostrado fundamentais para seu avanço (MOURA, 1999). Estes sistemas combinam software e hardware. Tais tecnologias permitem medir, controlar e gerenciar as operações logísticas. 
Por conseguinte, o sistema garante os ganhos de informações e controle sobre a operação são sem precedentes numa operação logística tradicional. A implantação eleva a empresa a um nível de maturidade corporativa muito grande e possibilitando a base para automações necessárias no atual cenário mundial.

\section{ESTUDO DE CASO}

A Bomix revoluciona o mercado nacional de embalagens plásticas ao lançar os baldes plásticos, solucionando finalmente o aproveitamento do espaço no armazenamento, transporte e design. Hoje, a empresa, líder no setor de embalagens plásticas no Brasil, possui o maior parque fabril da América Latina na produção de baldes industriais em cores e formatos diversos além de uma avançada central de logística e distribuição das embalagens. Para garantir entregas rápidas e eficientes em todo país, dispõe-se também de uma transportadora própria, a Bomixlog.

Nesse contexto, foram desenvolvidas técnicas e procedimentos para a melhor execução dos planos de trabalho, dentro do prazo adequado para procedimento das tarefas logísticas, assim abaixo, segue a fases de planejamento, desenvolvimento, verificação e otimização do projeto.

PRIMEIRO MÊS - Mapeamento das atividades, assim como levantamento das necessidades iniciais de melhoria do fluxo de transformação.

SEGUNDO MÊS - Análise de todos os processos operacionais. Cronoánalise e redesenho do processo de transbordo. Levantamento do índice de capacidade instalada. Projeto de conferência de movimentação do Porta-Pallete.

TERCEIRO MÊS - Durante o mês de setembro fizemos análises e acompanhamentos de todas as etapas que integram o centro de distribuição. Em busca de uma maior organização e melhoria de fluxo para atendimento do setor industrial, foi realizada uma proposta de sistema de identificação (Kanban), que está sendo analisada, discutida e aperfeiçoada pela equipe de logística. Para a melhoria do processo de transbordo, revisamos o relatório do controle de recebimento e atualizamos as informações do mesmo, para que, a partir desse novo relatório, seja possível identificar os tempos de cada operação e comparar com o estipulado como tempo padrão de operação. Para controlar as cargas paletizadas, desenvolvemos um novo relatório para registro de produção. Identificamos os registros sistémicos de entrada versus saída do centro de distribuição nos últimos 19 meses. Todos os processos e etapas ainda estão em desenvolvimento e serão concluídas dentro do prazo estipulado no plano de trabalho e atividades.

QUARTO MÊS - Análise de todos os processos operacionais. Levantamento do índice de capacidade instalada; Controle do recebimento (chegada de produtos - transbordo); 
Desenvolvimento e acompanhamento diário de KPI's (Fator de Utilização do Porta Pallet e Drive In); Participação de reuniões diárias de start up. Pesquisa de dispositivos de armazenamento; Controle do processo de palletização (falta de produção); Projeto de layout de instalação (armazenamento); Treinamento e desenvolvimento do sistema WMS; Redesenho do processo de transbordo e melhoria do fluxo de recebimento; Pré-Projeto de Kanban de Abastecimento.

QUINTO MÊS - Análise de todos os processos operacionais; Levantamento do índice de capacidade instalada; Controle do recebimento (chegada de produtos - transbordo); Desenvolvimento e acompanhamento diário de KPI's (Fator de Utilização do Porta Pallet e Drive In); Pesquisa de dispositivos de armazenamento; Controle do processo de palletização; Treinamento e desenvolvimento do sistema WMS; Levantamento do índice de máximo e mínimo de capacidade instalada e recebimento e expedição.

SEXTO MÊS - Análise de todos os processos operacionais; Levantamento do índice de capacidade instalada; Controle do recebimento (chegada de produtos - transbordo); Desenvolvimento e acompanhamento diário de KPI's (Fator de Utilização do Porta Pallet e Drive In); Pesquisa de dispositivos de armazenamento; Controle da processo de palletização; Treinamento e desenvolvimento do sistema WMS; Levantamento do índice de máximo e mínimo de capacidade instalada e recebimento e expedição; Levantamento da capacidade de palletização diária; Acompanhamento do número das ordens de serviço; Levantamento do número de unitizadores disponíveis.

SÉTIMO MÊS - Análise de todos os processos operacionais; Levantamento do índice de capacidade instalada; Controle do recebimento (chegada de produtos - transbordo); Desenvolvimento e acompanhamento diário de KPI's (Fator de Utilização do Porta Pallet e Drive In); Pesquisa de dispositivos de armazenamento; Treinamento e desenvolvimento do sistema WMS; Levantamento do índice de máximo e mínimo de capacidade instalada e recebimento e expedição; Acompanhamento do número das ordens de serviço; Levantamento do número de unitizadores disponíveis.

OITAVO MÊS - Análise de todos os processos operacionais; Levantamento do índice de capacidade instalada; Controle do recebimento (chegada de produtos - transbordo); Desenvolvimento e acompanhamento diário de KPI's (Fator de Utilização do Porta Pallet e Drive In); Pesquisa de dispositivos de armazenamento; Treinamento e desenvolvimento do sistema WMS; Levantamento do índice de máximo e mínimo de capacidade instalada e recebimento e expedição; Acompanhamento do número das ordens de serviço; Levantamento do número de unitizadores disponíveis; Elaboração das instruções de trabalho, especificando os meios e modos de operação do recebimento, armazenamento, movimentação e expedição; Fluxograma de todas as atividades 
industriais, assim como o layout de processos destas áreas; Participação do inventário, realizando conferência dos itens armazenados e registro das contagens dos materiais observados na base sistêmica e organização de materiais no armazém; projeto de layout da área de armazenamento dos itens recebidos (stage), otimizando sua região; Registro e controle dos produtos armazenados e expedidos do Porta-Pallet.

NONO MÊS - Análise de todos os processos operacionais; Levantamento do índice de capacidade instalada; Controle do recebimento (chegada de produtos - transbordo); Desenvolvimento e acompanhamento diário de KPI's de armazenamento, transbordo, expedição, consumo de produtos; Pesquisa de dispositivos de armazenamento; Treinamento e desenvolvimento do sistema WMS; Levantamento do índice de máximo e mínimo de capacidade instalada e recebimento e expedição; Revisão da instrução de trabalho da expedição/faturamento; Projeto de layout da área de armazenamento dos itens expedidos (stage), otimizando sua região; Registro e controle dos produtos armazenados e expedidos do Porta-Pallet; Auditoria do sistema de Gestão da Qualidade.

DÉCIMO MÊS - Análise de todos os processos operacionais; Levantamento do índice de capacidade instalada; Controle do recebimento (chegada de produtos - transbordo); Desenvolvimento e acompanhamento diário de KPI's (Fator de Utilização do Porta Pallet e Drive In); Pesquisa de dispositivos de armazenamento; Levantamento do consumo de filme stretch por pallet produzido; Cronoanálise do processo de palletização; Levantamento do índice de produção vs expedição; Registro e controle dos produtos armazenados e expedidos do Porta-Pallet através das movimentações realizadas; Levantamento dos itens de prioridade para o armazém (pipeline); Projeto de ampliação da capacidade de produção de pallets.

DÉCIMO PRIMEIRO - Análise de todos os processos operacionais; Levantamento do índice de capacidade instalada; Controle do recebimento (chegada de produtos - transbordo); Desenvolvimento e acompanhamento diário de KPI's (Fator de Utilização do Porta Pallet e Drive In); Pesquisa de dispositivos de armazenamento; Cronoanálise do processo de palletização; Registro e controle dos produtos armazenados e expedidos do Porta-Pallet; Projeto de similaridade de produtos; Projeto de receita por região.

DÉCIMO SEGUNDO MÊS - Análise de todos os processos operacionais; Levantamento do índice de capacidade instalada; Controle do recebimento (chegada de produtos - transbordo); Desenvolvimento e acompanhamento diário de KPI's (Fator de Utilização do Porta Pallet e Drive In); Pesquisa de dispositivos de armazenamento; Treinamento na base sistêmica da produção; Enceramento de ordens de produção; Participação do processo de auditoria ISO 9001; Desenho de layout para área de produção de pallets (produto acabado). 


\section{DISCUSSÃO}

Através da observação e, por conseguinte, a estruturação de modelos adequados de produção enxuta, as bases do gerenciamento da cadeia de suprimentos dentro do armazém foram sustentadas através dos processos de pesquisa e desenvolvimento dentro do cronograma especificado, em face com as demandas da produção e mercado.

As atividades logísticas absorvem uma parcela relevante dos custos totais, representando em média, dessa forma, para o sucesso no processo logístico foi muito importante a incorporação de um sistema de informações que possa atender e dar suporte a todos os processos que compõem sua estrutura. Dentro da administração de materiais, o planejamento da produção, o suprimento e a distribuição física integraram-se para remodelar o gerenciamento dos recursos fundamentais.

As medições e posteriormente criação de indicadores de desempenho foram o ponto primordial para direcionar o progresso do projeto, a vista que a organização não apresentação nenhuma forma de medição dos resultados, assim, houve um suporte ao desempenho das atividades primárias, para que a empresa pudesse demonstrar evolução em toda cadeia logística. Em paralelo, a padronização das atividades contribuiu para organizar e distribuir todas as funções e operações do armazém, dispondo, assim, as necessidades fundamentais e urgentes, abrangendo a administração dos espaços necessários para manter os materiais. Essa atividade é muito relevante, pois diminuiu a distância entre as operações, além de envolver diversos processos como: localização, dimensionamento, recursos materiais e patrimoniais (arranjo físico, equipamentos, etc.), pessoal especializado, recuperação e controle de estoque, embalagens, manuseio de materiais, montagem/desmontagem, fracionamento e consolidação de cargas e consequentemente a necessidade de recursos financeiros e humanos. Em consonância com os resultados, observa-se o desenvolvimento técnico-profissional dos operadores, que foram peço fundamental para o seguimento do projeto, na qual o treinamento proporcionou a evolução das técnicas, disseminação das mesmas e consolidação das ferramentas disponíveis.

O sistema de tecnologia da informação aplicado foi importante em todo estágio da cadeia de suprimentos, pois permitiu que a organização analisasse as informações que são necessárias para a tomada de decisão, sendo segmentadas de acordo com os estágios da cadeia de suprimentos e seus diferentes níveis de funcionalidade que podem receber e apresentar informações diferenciadas e analisá-las para solucionar problemas a curto ou a longo prazo, assim, utilizado para tomar decisões de estratégia, planejamento ou operação.

Deste modo, o armazém pôde melhor desempenhar vários papéis dentro da estrutura de 
distribuição adotada pela empresa, que tange a recepção e consolidação de produtos de vários fornecedores, para posterior distribuição a diversos canais de distribuição de uma rede e recepção de produtos da fábrica e distribuição para diversos clientes.

\section{CONSIDERAÇÕES FINAIS}

Através da execução do plano de trabalho, tal projeto apresenta aspectos competitivos na qual contribuiu para o crescimento acentuado da organização no que diz respeito ao gerenciamento da cadeia logística, assim como mecanismos de desenvolvimento competitivo, necessário para garantir a manutenção de sua existência no mercado.

Dessa forma, o novo cenário logístico visa atribuir aos processos uma maior competência no que tange aos resultados e na relação com os clientes e fornecedores. É importante observar que neste contexto as ferramentas de tecnologia da informação, gestão da cadeia de suprimentos, gerenciamento da produção e planejamento, controle e programação da produção contribuíram efetivamente para oferecer ao sistema logístico o efetivo desempenho esperado para todas as etapas do processo produtivo e distribuição da organização.

Entre os processos logísticos, é possível destaca as questões relacionadas à gestão dos pedidos, dos sistemas de transporte e os mecanismos de armazenagem de produto intermediário e acabado, na qual a sua evolução proporcionaram o aumento da disponibilidade de recursos frente às demandas, pois o bom controle do armazém permitiu o rápido resgate de materiais e a gestão eficiente dos recursos.

A partir dos indicadores gerados diariamente e mensalmente, foi possível identificar divergências no processo/serviço, proporcionando uma agilidade na verificação do erro e correção, auxiliando nas tomadas de decisões gerenciais, assim como as tomadas de ações corretivas e preventivas a possíveis anomalias identificadas nos resultados verificando as funcionalidades, ganho e dificuldades com o processo como um todo.

Como sugestões para estudos futuros é interessante a medição da eficiência de dispositivos de carregamento e expedição que contribuem para o armazenamento agilizado; A identificação da redução o tempo de espera dos produtos dentro do CD; Os impactos da inclusão de novas atividades junto a incorporações do sistema de informação. 


\section{REFERÊNCIAS}

APTE, Uday M.; VISWANATHAN, S. Effective cross docking for improving distribution efficiencies. International journal of logistics: research and applications. 2000

CALAZANS, Fabíola. Centros de distribuição. Gazeta Mercantil. 2012.

GIANESI, I. G. N.; BIAZZI, J. L. Gestão estratégica de estoques. R.Adm., São Paulo, 2011.

LIMA, Maurício P. Armazenagem: considerações sobre a atividade de picking. Centro de Estudos em Logística (CEL), COPPEAD/UFRJ. 2012

MOURA, R. A. Kanban: a simplicidade do controle da produção. São Paulo: IMAM, 1996.

MOURA, Reinaldo A. Sistemas e técnicas de movimentação e armazenagem de materiais. 4a ed. São Paulo: IMAM, 452 p. 2008

TOMPKINS, J. A. Facilities planning. 2a ed. 1996 